\title{
Title: Bioengineering methods for organoid systems
}

Authorship: Jad Saleh+, Barbara Mercier ${ }^{+}$, Wang XI*

Affiliations and Addresses: Université de Paris, CNRS, Institut Jacques Monod, Paris, France

${ }^{+}$Authors contributed equally to this work.

* Corresponding Author. Correspondence to wang.xi@ijm.fr.

Key Words: stem cell behavior, designer biomaterials, cellular microenvironment, biotechnologies, organ-on-a-chip 


\section{Abstract}

Organoids have been widely used in fundamental, biomimetic and therapeutic studies. These multicellular systems form via cell-autonomous self-organization where a cohort of stem cells undergo in vivo-like proliferation, differentiation and morphogenesis. They also recapitulate a series of physiological cell organization, complexity and functions that are untouchable by conventional bio-model systems using immortal cell lines. However, the development of organoids is often not easily controlled and their shape and size are yet fully physiological. Recent research has demonstrated that multiple bioengineering tools could be harnessed to control important internal and external cues that dictate stem cell behavior and stem-cell based organoid development. In this review, we introduce the current development of organoid systems and their potentials, as well as their limitations that impede their further utility in research and clinical fields. In comparison to conventional autonomous organoid system, we then review bioengineering approaches that offer improved control over organoid growth and development. We focus on the genetic editing tools that allow the program of build-in responses and phenotypes for organoid systems with enhanced physiological relevance. We also highlight the advances in bioengineering methods to modify cellular external milieus to generate desirable cell composition, 3D micro-architectures and complex microfluidic systems. We conclude that the emerging biomimetic methods that employ multidisciplinary approaches could prevail in the future development of organoid systems.

\section{Introduction}

Modeling biological processes and diseases in vitro has been the basis of biomedical research. It allows scientists to recapitulate physiological conditions and body functions from the molecular to the cellular, tissue, and organ level. There are advantages in using animal models, since they are in vivo models that have close physiological similarities to human. However, these models pose many disadvantages such as the biological differences between animals and humans, difficulty in accessing tissues for imaging, costly and time-consuming procedures in generating genetic clones, and limited throughput ${ }^{1}$. On the other, for decades, immortalized cell lines cultured on two-dimensional (2D) substrates have been the gold standard for in vitro studies of certain organs or tissues, owing to their simplicity, reproducibility, cost-effectiveness, and potential for long-term culture. Such cell lines are 
either derived from naturally-occurring carcinomas that keep dividing or have been genetically altered to proliferate indefinitely and be cultured for prolonged periods of time. Countless number of cell lines have been developed, each characterized by their own phenotype depending on the tissue from which they were derived. One big disadvantage of such cell lines is the fact that they undergo mutations to become immortal and can further acquire genetic variations after many passages, thus are not able to completely recapitulate the cell types found in vivo ${ }^{2,3}$. Another drawback of conventional 2D cell culture methods is that they lack the conditions that allow for natural three-dimensional (3D) cellular organization. This significantly affects important cell signaling networks, making 2D systems ineffective for accurate drug screenings ${ }^{4}$. Additionally, since these cell lines are made of a single cell type, the cell-cell interactions of different cell types are not fully present in these in vitro systems, thus failing to recapitulate certain in vivo signaling pathways. Alternatively, culture methods using stem cells are promising for studying tissue morphogenesis, pathologies, developing new stem cell therapies through drug screenings, and applications in regenerative medicine. However, until recently, conventional stem cell culture systems on 2D substrates relied on culturing specific stem cells or stem cell-derived cell types ${ }^{5}$, which lacked the differentiated cell types found in the native tissue. These systems are unable to provide the proper conditions needed to maintain and expand the stem cell population while at the same time ensuring its potential to differentiate into its different lineages.

As the concerns for the limitations of $2 \mathrm{D}$ culture accumulated, researchers devoted their efforts in revealing the roles of extracellular matrix (ECM) in offering important 3D biochemical and biomechanical cues for tissue homeostasis and development. For example, previous works at the end of last century had demonstrated that ECM contributes morphogenic signals during mammary gland branching morphogenesis ${ }^{6}$ and disturbing the interactions between gland epithelial cells and ECM causes improper gland development and tumor formation? ${ }^{7}$. Such studies illustrated the influence of 3D microenvironment for tissue morphogenesis and the need for cells to be exposed to a physiological exogenous matrix to maintain their differentiated state. Indeed, Emermen and Pitelka showed that on floating collagen membranes, epithelial cells could maintain their ability of secreting milk proteins as differentiated cells ${ }^{8}$. When the same cells were cultured on 2D plastic culture dishes, they lost this ability even in the presence of lactogenic hormones. Similarly, Bissell and colleagues 
demonstrated that, unlike monolayer cultures, human mammary epithelial cells within 3D laminin-rich matrices (Matrigel) self-organized into acini-like structures that recapitulated several features of in vivo architectures ${ }^{9}$. In this context, 3D culture led to the development of normal and malignant tissues from distinct cell sources, whereas 2D surfaces led to practically indistinguishable phenotypes ${ }^{9}$. These findings allowed scientists to use 3D cultures to decipher the morphogenic processes and the biological activities that cause tumor progression ${ }^{10-13}$. For instance, using a 3D model system of growth-arrested human mammary epithelial cells, Bissell and Brugge were able to identify genetic abnormalities associated with the early stages of carcinogenesis in culture ${ }^{12}$. They demonstrated that the activation of an epidermal growth factor receptor, ERBB2, in MCF-10A cells during morphogenesis leads to abnormal 3D epithelial architectures with the presence of a multiacinar phenotype. This was attributed to an ability to escape proliferative suppression and the loss of normal apoptotic activity, which are hallmarks of cancer ${ }^{12}$. In a following work, such hyperproliferative phenotype induced by oncogenes such as ERBB2 and RAS was found to depend on PI3K activity and only seen in 3D systems, not in $2 \mathrm{D}$ culture ${ }^{13}$. All these findings pinpointed the importance of ECM in guiding in vitro tissue morphogenesis so as to enable better mimicking in vivo conditions. The development of 3D ECM culture techniques later laid a key foundation for the emergence of organoid culture.

In parallel to the progression of $3 D$ culture techniques, investigations in embryonic development started to unravel the profound mechanism underlying organogenesis. Additionally, studies of key signaling pathways in stem cell niches of different organs have offered insights into how they are able to control stem cell maintenance and differentiation. Profiting from this knowledge, researchers supplemented 3D culture approaches with essential growth signaling molecules and this helped kick-start the modern field of organoids - the stem cell-derived 3D culture systems that are able to sustain self-renewal, generate multiple cell types, and allow for the spatial self-organization of cells similar to native organs (Figure 1). Starting from the seminal works by Sasai ${ }^{14}$, Clevers $^{15}$, and colleagues, organoids are commonly developed in a 3D microenvironment of Matrigel that mimics ECM and so far, various organoids have been derived from organ-specific adult stem cells (ASCs), induced pluripotent stem cells (iPSCs) and embryonic stem cells (ESCs). Unprecedented advantages in better emulating in vivo features compared to classical 2D cultures have earned these systems 
broad recognition in many fundamental and translational biology studies ${ }^{16}$. Indeed, 3D organoids represent faithfully the tissue homeostasis and organ-specific organogenesis. They harness stem cells as the "draft horse" to enable proliferation and differentiation of multiple, tissue-specific cell lineages. Within the 3D culture environment, these different progenies are able to self-organize into different compartments reminiscent of the in vivo organization. Furthermore, such complex structures recapitulate physiological cell polarization, cell-cell interaction, cell-matrix contacts and even certain functions that match what is seen in native tissues. Hence, organoid systems fill in the gap between in vivo and in vitro for accurate studies of many biological processes, such as morphogenesis, stem cell niche biology, tissue homeostasis, and the effect of genetic mutations and drugs.

Although using organoid systems has significantly improved our biomimetic platforms, they still possess a number of limitations, which have hindered the full realization of their potentials. One of those is the lack of generic cells such as endothelial, nervous and immune cells, as well as other types of tissues present in the physiological microenvironment ${ }^{17}$. Without these supportive cells and proper vascular networks for the nutrient delivery and waste removal, it is difficult to increase organoid size, lifetime, maturation and complexity. Also prevalent is the problem that many organoids possess an enclosed and physically inaccessible central lumen. Another complication that arises from current organoid culture is the use of Matrigel as a culture matrix, which is made from mouse sarcoma cells and is known for its batch-to-batch variability. Moreover, organoids do not possess regularly patterned structures as their native tissues in that they are generally allowed to grow freely without the physical and chemical constraints and guidance found in vivo. Deriving from such an uncontrolled, cell-autonomous approach, most self-organized organoids present shape heterogeneity, low reproducibility, variability in stem-cell differentiation outcomes and celltype(s) composition. These major disadvantages have significantly prevented generating higher-grade organoids and gaining control of their functionalities. Moreover, under the framework of autonomous organization, it is hard to conclude from experimental results whether they are real phenotypes or merely intrinsic inconsistencies due to chances, thus hampering many applications. Therefore, solutions that overcome these major drawbacks of this powerful culture model are currently highly sought-after. 
The convergence of bioengineering and organoid biology has started to get a grip on key factors that underpins stem cell behavior, tissue homeostasis and cell-cell/cell-environment interactions in organoid systems. The main purposes of such interdisciplinary efforts are focusing on using engineering solutions to harness numerous intrinsic and extrinsic cues to direct the growth, development, organization, and architecture of organoids. The delicate control over intrinsic sensing pathways, genetic information of the cells, tissue-tissue interfaces, rigidity of the substrate, geometry, systemic stress and vascular components ${ }^{18-20}$ has enabled one to dictate stem cell fate and guide the organization of their progenies in an organoid. These discoveries fostered the notion that the development of artificial organoid systems could be designed and guided by various bioengineer strategies to further enhance their utility and physiological relevance ${ }^{19}$ (Figure 1). Particularly, in the past decade, we have witnessed a surge in the development of novel biomimetic approaches for organoid studies aiming either to discover the fundamental biological principles or to revolutionize the existing systems for tissue engineering ${ }^{18-20}$. In this review, we compare the conventional system exploiting cell-autonomous organization and bioengineering guided organoid formation. By introducing the basic of organoid systems, we pinpoint the numerous and often entwined factors that dictate organoid growth but are currently poorly controlled. We also discuss how advances in bioengineering methods could provide pre-defined cues to steer the complex organoid organization and leverage the heterogeneous and stochastic nature of organoid development. Finally, we envision that by mastering and carefully integrating bioengineering methodologies, researchers could overcome the challenges in regulating the autonomous self-assembly capacity of stem cells and further the limits of in vitro organogenesis to acquire desirable organoid systems.

\section{Permissive autonomous self-organized organoids}

At present, many conventional organoid systems employ "permissive mode" of organoid generation. This means that they main rely on cell-autonomous 3D culture techniques that take advantage of the self-organization responses of ASCs of a specific tissue or pluripotent stem cells (PSCs). With minimal exogenously provided controls, such methods "permit" the intrinsic properties of the cells to drive the formation of various organoids. For instance, in their seminal work in 2008 Clevers and colleagues ${ }^{15}$ observed the growth of Matrigel- 
suspended intestinal crypts, which house LGR5+ stem cells adjacent to specialized Paneth cells from mouse or human tissues. They discovered that under the stimulations of small molecules such as EGF, Noggin, and R-Spondin1, these crypts could self-organize into cysts with a central lumen and in the next few days projected irregular protrusions (budding), characterized by the presence of LGR5+ stem cells, Paneth cells, and transit-amplifying cells, the main cell types of intestinal stem cell niche. Such multiple "crypt fission events" are companied simultaneously with the self-formation of villus-like epithelia ${ }^{15}$, which also face the lumen and contain all differentiated intestinal cell types, i.e., enterocytes, and tuft, enteroendocrine, goblet and $\mathrm{M}$ cells. In short, these intestinal tissue-resembling organisms, or intestinal organoids ("mini-guts"15) were emerged by the orchestrated effects of proliferation, differentiation, organization and migration of intestinal stem cells (ISCS) in a cell-autonomous manner. The lineage potential and the stage of stem cells are then major determinants of organoid development. As such, ASC-derived organoids reflect closely the adult tissue homeostasis and renewal processes and are restricted to certain kind of tissue. Until now, with relentless efforts organoids have been generated from various individual tissues, including pancreas ${ }^{21}$, liver ${ }^{21}$, prostate $^{22}$, and stomach $^{23}$ (Figure 1). Alternatively, under coordinated regulation, PSC's broad potency would allow one to obtain multiple lineages, reflecting various types of tissues. For example, the PSC-originated intestine ${ }^{24}$, gastric ${ }^{25}$ and lung ${ }^{26}$ organoids contain one co-evolved mesenchymal cell layer. This property adds a level of organ-like complexity to the PSC-originated organoid development and makes them an excellent candidate for the study of tissue-tissue crosstalk ${ }^{20}$. Hence, PSC may represent the optimal starting point for generating various organ ontogeny models and early stage embryonic systems to study the relative contribution of many cues and molecular factors to a complicated morphogenetic process. This has been successful as exemplifying by numerous PSC-derived organoids, including intestine ${ }^{27}$, brain ${ }^{28}$, kidney ${ }^{29}$, liver ${ }^{30}$, lung ${ }^{31}$, blood vessel ${ }^{32}$, and optic $\operatorname{cup}^{33}$ (Figure 1). Although PSC-derived organoids employ self-assembly at the pluripotency stage, it often requires the set-up of complicated culture conditions to limit the developmental variations and guide differentiation ${ }^{34,35}$. In case the organoid development becomes unpredictable, it could result in uncontrolled growth of tissue aggregates as exemplified in the formation of optic cups with random number of vesicles per aggregate ${ }^{33}$ and stomach organoids ${ }^{36}$ that are embedded inside an arbitrarily distributed cell mass. 
The advantages of organoids include incomparable complexity in terms of architecture and function by recapitulating the different population of cells in tissues ${ }^{16,37}$, versatility and relative simplicity of culture as well as the compatibility with many existing biological tools. These have made them an excellent biomimetic proxy for health and disease tissue development studies (Figure 1). Under a permissive condition, the formation of organoids by a group of stem cells include complex processes of self-sorting, self-patterning and selfmorphogenesis ${ }^{38}$. These processes reflect largely how intrinsic cell properties response to and regulate time-evolving local microenvironment and result in the control of relative cell positions, regulation of different cell status and emergence of an ordered organization via intrinsic tissue mechanics constraints. Hence, such systems may offer clues to long-standing matter of intrigue and interest in developmental biology. For instance, the interplay between Wnt-activated Lgr5 stem cells and Paneth cells is the key to self-renewal of gut organoids ${ }^{39}$. Their physical association in vitro resembles their interdependence in vivo and reflects intestinal crypt self-sustaining mechanism. Additionally, other morphogenetic mechanisms, such as the self-organization of optic-cup from homogeneous progenitors ${ }^{33}$ and forebrain tissue formation guided by long-range signaling ${ }^{40}$, have also been revealed using organoid systems. Following this line, organoids have also been extensively used to model human disease development, such as cancer ${ }^{41}$ and cystic fibrosis ${ }^{42}$, and to develop personalized regenerative medicine ${ }^{42}$. A representative example is the establishment of cancer organoids (tumoroids) from different patient biopsy samples (e.g. stomach ${ }^{43}$, $\operatorname{liver}^{44}$ and $\operatorname{colon}^{41}$ ). These tumoroid models not only recapitulate the molecular fingerprints of their origins, but also show high morphological similarity in the histological subtypes and differentiation phenotypes ${ }^{45}$. Additionally, architectural and genetic heterogeneity of in vivo tumors are also highly preserved by tumoroids that are self-organized by cell sorting ${ }^{44}$. These features allow direct investigation on the functional, developmental and metabolic characteristics as well as drug responses of cancer-derived tissues. In a recent work by Broutier et al. ${ }^{44}$, liver tumoroids have been used for drug-screening test and provided the proof-of-principle of the therapeutic potential of the ERK inhibitor SCH772984 in treating liver cancer. In addition, PSC-derived liver organoids have offered new opportunities in investigating liver fibrosis and toxicity ${ }^{46}$. In another work, Bartfeld and colleagues ${ }^{43}$ established healthy and cancerous gastric organoids and infected them with $\mathrm{H}$ pylori. In this way, they identified the activation of the nuclear factor-kB pathway and up-regulated gene human chorionic gonadotropin $\beta$, which are 
associated with stomach cancer ${ }^{47}$. Moreover, although there are still major hurdles to overcome to use organoids for regenerative medicine, pioneering studies, such as organoids from renal cancer patients ${ }^{48}$, have started to pave the way to orthologous organ replacement ${ }^{30,42}$ and reduction in the use of animal models ${ }^{45}$ for ethical reasons. The recent promising breakthroughs in this direction has been extensively reviewed by multiple groups ${ }^{49-}$ 51.

As organoids are approaching their initial destination of modeling healthy and pathological phenotypes, problems associated with cell-autonomous self-organization also emerged. Firstly, this self-assembly process as mentioned above normally leads to organoids with a physically inaccessible lumen. This makes it difficult to access the apical part of the tissue in intestinal organoids for example, where the differentiated surface analogous to the intestinal cavum for nutrient absorption is facing a sealed off lumen (Figure 1). Such a configuration does not allow shedding dead cells either, resulting in a necrotic core that limits organoid size and disturbs long-term imaging. It also prevents direct probing tissue responses to other exogenous stimuli that interact with the in vivo luminal epithelial surface, such as metabolic enzymes and microbial communities ${ }^{52}$. Although the polarity of conventional enteroids could be everted to an apical-out polarity by removing ECM scaffold proteins ${ }^{53}$, such an approach reduces the percentage of proliferating cells in the organoids, thus raising doubts about their capability of self-renewal and long-term culture of the system. Besides, the autonomous approach often fails to develop organoids consisting of generic cell types and tissue-tissue interfaces. Using self-organization method, this problem is partially mitigated by co-culture of cells sharing different origins. For instance, co-culture of human iPSC-hepatic endoderm cells, umbilical vein endothelial cells (HUVECs) and mesenchymal stem cells (MSCs) could generate liver buds consisting of endothelial networks ${ }^{30}$. The introduction of human blood vessel organoids ${ }^{32}$ may also trigger the formation of perfused vascular trees between avascular organoids. Similar co-culture approaches have been applied to create neural/intestinal ${ }^{54}$, hepatic stellate cells/hepatocytes ${ }^{46}$ and tumor/immune cells ${ }^{55}$ interfaces in organoids. Nevertheless, these co-culture systems still fail to reproduce organ-like highorder of structural and cellular sophistication. Furthermore, the permissive systems are lacking regulative factors that could limit the inter-organoid variability in their relative cellular components, dimension and architectural features. Such variabilities due to iterative cell 
autonomous interaction with other cells and local chemical, biophysical cues lead to poor reproducibility and functional immaturity that hamper organoid's applications in biomodeling and potential for transplantation. Various efforts have been made and bioengineering methods have shown promising results in improving organoid systems.

\section{Bioengineering approaches for growth and development of organoids}

The emerging field of organoid systems has offered a promising platform to bridge the current significant gap between in vivo and in vitro. In addition, the study of organoids and the properties of stem-cell based self-organization bolsters the notion that with proper guidance that is lacking or poorly controlled with conventional culture methods, various functional organ tissues could be reproduced in a test tube ${ }^{20}$. However, current culture approaches that are fully relied on the autonomous assembly of stem cells has led to low reproducibility, uncontrolled cell type development, limited tissue size, maturity and life span, and organoids of partial components of native tissues ${ }^{19,20}$. These drawbacks have significantly impeded the realization of the full potential of organoids in fundamental biology, tissue engineering and regenerative therapies ${ }^{19}$. To overcome these challenges, researchers have found resort in bioengineering methods to provide precise control over intrinsic properties and cellular environment and guidance for the growth and development of organoids. Herein, we discuss the emerging strategies and highlight the latest bioengineering advancement to acquire desirable organoid systems.

\section{Customize intrinsic properties of stem cells}

Within the framework of self-organization, one key factor that determines the characteristics of organoids is the intrinsic stem cell properties, such as the lineage potential and the stage of stem cells. These genetic properties of stem cells may be expanded and modified by the recent advances in biotechnology for direct manipulation of endogenous genes through CRISPR or TALEN technology. The genetic modification could trigger novel changes in stem cell behaviors and then results in user-defined organoids accordingly (Figure 2A). A seminal 
work by Schwank et al. ${ }^{42}$, has demonstrated that organoids developed from intestinal stem cells (ISCs) of cystic fibrosis patients could be reverted to normal organoids via CRISPR/Cas9 editing. The researchers targeted single-gene mutation by deleting phenylalanine at position 508 and generated intestinal-organ-like units for autologous transplantation back into the patients for tissue repair and functional restoration. Such genome editing approach then offers a new possibility to generate sources of tissue for autologous therapy ${ }^{56}$. Alternatively, transcription factor expression could be modified to genetically guide morphogenesis events $^{57}$. A transient pulse of GATA-binding protein 6 expression induced by genetic engineering has led to the development of multiple germ layers and liver bud-like phenotype ${ }^{58}$. These genetic tools also significantly extend our sources of stem cells. In the case of patient-derived organoids for example, the direct establishment of organoid-based disease models could be difficult when the disease itself is ultra-rare or due to lethal genetic defects before birth ${ }^{45}$. Using gene-editing technologies, such as lipofection-based transfection $^{59}$, lentiviral infection ${ }^{60}$ and electroporation ${ }^{61}$, to induce mutations of specific genes $^{62,63}$ (Figure 1), various disease models due to genetic and epigenetic aberrations can be reconstructed in the form of organoids. Furthermore, cell-cell interactions and cellular behaviors can be modulated by modifying the cell membrane with $D_{N A}^{64,65}$, liposomes ${ }^{66}$ and aptamers $^{67}$ to regulate cell adhesive properties (Figure $2 \mathrm{~A}$ ) and with growth-factor loaded nanoparticles to control autocrine signaling loop ${ }^{68}$ (Figure 2A). On a DNA-patterned template, cells functionalized with short and degradable oligonucleotides can reversibly adhere into arrays in a modular fashion ${ }^{64}$. Hybridization of complementary DNA then further allow the assembly of DNA-coated cells with user-specified cell-cell contacts, resulting in robust cell sorting and tissue-tissue interfaces during the building of 3D microtissues ${ }^{65}$. Thus, such a treatment could be applied to modify the cell membranes of different stem cells to offer promising control over organoid self-organization processes. For instance, the formation of Rathke's-pouch-like tissue structures occurs at the interface of non-neural head ectoderm and hypothalamic neuroectoderm ${ }^{69}$. To produce such defined tissue-tissue interfaces that are important for the self-driven morphogenesis, desirable cellular interconnectivities could be modulated by cell membrane-modification, leading to artificial cell sorting with controlled layer formation. Moreover, for autologous transplants therapeutic stem cells could be surface-engineered and harvested in the form of organoids to endow the donor cells specific adhesive properties to engage targeted cells or tissues ${ }^{67,68}$. 


\section{Synthetic hydrogels for organoid studies}

In conventional organoid systems, the organization of the cell mass is highly stochastic and its cell-type composition highly heterogeneous. For example, in intestinal organoids, crypts are random oriented and the length and number of crypts can vary considerably from one organoid to another. Such a lack of reproducibility is mainly due to uncontrolled stem cell proliferation and differentiation in a 3D matrix. Recent investigations have shown that stem cell behaviors are controlled by its external microenvironment ${ }^{70}$ and the properties of the matrix ${ }^{18}$. The cell-substrate interplay via cell adhesive properties allow stem cell to sense the stiffness, nanotopography, geometry and chemistry of ECM. This together with cell-cell interaction and soluble biochemical cues (see below) in space and time leads to dynamic sensing signaling cascades that regulate stem cell fates and self-assembly. Thus, the biomaterials used for organoid culture should provide essential in vivo cues and means to control these cues for the growth of organoids.

The most commonly used substrate for growing organoids is Matrigel, a gelatinous protein mixture extracted from Engelbreth-Holm-Swarm mouse sarcoma cells ${ }^{71}$ via decellularization of native tissues. The aim of such a process is to remove cellular component from the tissue by mechanical, chemical and enzymatic means while reserve the ECM compositions, such as chemical components and ultrastructure ${ }^{72,73}$, resulting in biocompatible scaffolds. Such ECMderived bio-scaffolds have been produced in the forms of hydroge ${ }^{71}$, including Matrigel and collagens, dry sheets ${ }^{74}$, or powders ${ }^{75}$ and have been shown to support stem cell growth and self-assembly. Another class of native-derived ECM consists of scaffolds made from decellularized organ tissues that preserve both the structural and functional integrity ${ }^{76}$. Using cells of the same origins, it is possible to repopulate the organ-specific ECM scaffolds. For instance, in an attempt to form intestinal epithelial barriers in vitro decellularized gut matrix was capable to support ISCs expansion and differentiation, as well as the co-culture of gutderived fibroblasts ${ }^{77}$. However, ECM of natural sources is inherently heterogeneous, resulting in high batch-to-batch variation and potential xenogeneic contamination ${ }^{78,79}$, which could further contribute to the variation in organoid development. Moreover, these materials are normally weak in mechanical properties and their multiple components lack instructive 
spatiotemporal distribution. This impedes the reproduction of elaborate morphogenesis that is guided by highly regulated cues in vivo. These challenges can be addressed by the development of synthetic elastomeric matrices with defined compositions and properties. Multiple variables, such as adhesive ligand density, crosslinking degree, material viscoelasticity and topography, porosity, as well as degradability, are controllable by modern synthetic chemistry approaches ${ }^{80,81}$. For example, inert poly(ethylene glycol) (PEG) hydrogels could be enriched with fibronectin, laminin, hyaluronic acid, collagen, perlecan and RGD (ArgGly-Asp) peptide to mimic essential in vivo biochemical signals. Degradable PEG gels could be made by incorporating hydrolytically or enzymatically labile segments to offer dynamic mechanical properties ${ }^{82}$. In a recent attempt, a four-arm poly(ethylene glycol) (PEG) hydrogel with maleimide terminus and tunable mechanical properties was prepared and functionalized with adhesive RGD peptide in a co-polymerization procedure ${ }^{83}$. This synthetic matrix was able to support reproducible human intestine and lung organoid development in vitro. The hydrogel can also act as delivery vehicles of organoids for engraftment at implantation sites, avoiding the risks of immunogen and pathogen transfer posed by natural tumor-derived ECM matrices.

Besides PEG, other promising materials for producing defined matrices include polyacrylamine (PAA), and natural macromolecules, such as alginate, agarose, fibrin, and collagen ${ }^{19,20}$. The exquisite tuneability of their material properties enables systematic variations and test with robotic micropatterning techniques ${ }^{84,85}$. One could produce large arrays of hydrogels with different elasticity, porosity, degradability, and chemical compositions for the evaluation of the influence on stem cell behaviors. From such highthroughput analysis on multiple parameters, researchers could extract the information about the most suitable biomaterial combination for modulating organoid in a desired manner. For instance, synergistic or antagonistic effects amongst degradability, biochemistry, rigidity and cell density on ESC self-renewal capacity in hydrogels had been unraveled using 3D liquiddispensing technology ${ }^{84}$. Furthermore, physiological topography cues down to nanometer scale could be added to synthetic bio-scaffolds as another layer of complexity using electrospinning, nanolithography, and selective etching. These technologies allow for the production of artificial substrates consisting of ultrafine fibers (by electrospinning), nanoscale features (i.e., nanowells, nanolines, nanopillars by nanolithography), and roughen 
surfaces (by etching). These topography features have been shown to impact profoundly on stem cell fate and behavior ${ }^{18}$. For example, nanopits and nanogratings could elicit changes in alignment, adhesion, and mechanical properties of human stem cells ${ }^{86,87}$, impacting differentiation, expansion, and self-renewal, thus providing artificial guidelines for the later development of organoids. Besides guiding stem cell behaviors, hydrogels were also produced in other formats for various applications. For example, a thermoresponsive hydrogel that transits from liquid to solid phase between $4{ }^{\circ} \mathrm{C}$ and $37{ }^{\circ} \mathrm{C}$ allows serial expansion and rapid retrieval of hPSCs for biomedical applications ${ }^{88}$. For scalable expansion and differentiation of PSCs, alginate beads ${ }^{89}$ and capsules ${ }^{90,91}$ that are loaded with stem cells were also developed to match production using industrial scale stirred tank bioreactors.

Nevertheless, in compared to native ECMs such as Matrigel, synthetic bioscaffolds are still in primitive stage as their major drawback is the usual lack of dynamic properties of native matrices for cell-driven remodeling. Synthetic hydrogels formed via stable, covalent crosslinks generally do not allow for broad matrix remodeling and fiber displacement ${ }^{92}$, that prevents in-vivo like morphogenesis processes and obtaining organoids of large size (millimeter scale). Hence, the future development of synthetic designer matrices for organoids would need to include bioresponsive materials ${ }^{80,81}$ that contain physical crosslinks and/or active bonds susceptible to proteolysis or enzymatic degradation. These components would allow relaxation and rearrangement in response to cellular forces and enzymatic cleavage, mimicking native ECM mechanics. For example, RGD, a generic cell adhesion peptide, could be coupled to long and flexible tethers in hydrogels that allow cells to reorganize and pull them into clusters ${ }^{93}$. To produce cell-degradable matrices, peptide-based cross-linkers that are susceptible to proteolysis and other enzymes such as plasmin, elastase and matrix metalloproteinases (MMPs) can be included in functionalized hydrogels ${ }^{94,95}$. The hydrogels containing synthetic peptides, which are recognizable and responsible to local cell-secreted protease levels, then enable cell-mediated point of clearage, promoting desire cellular behavior, such as directed migration ${ }^{96}$. In addition, bio-reactive hydrogels that mimic matrix stiffening during aging, development and disease have been developed by multiple approaches to study various collective cell mechano-sensing mechanisms ${ }^{97-99}$. Interestingly, another level of complexity in bioreactivity can be achieved by incorporation of multiple bioresponsive sites. By combining protease-degradable sites, cell-adhesion motifs, and 
growth factors in PEG for instance, sustained delivery of vascular endothelial growth-factor (VEGF) could be achieved during MMP-regulated hydrogel degradation, promoting vascularization in vivo ${ }^{100}$. Nevertheless, many of these materials haven't been applied to organoid studies. Furthermore, major hurdles in developing bioactive hydrogels, including controlling polymerized/depolymerized byproducts and gel degradation kinetics in biocompatible and bio-inert formats ${ }^{95}$, are still ongoing challenges to be conquered. Significant improvement and convergence of synthetic ECM and organoids in the future should lead to the generation of mimicking systems to study tissue homeostasis and pathogenesis.

\section{Bioengineering seeding conditions}

In vivo, diffusive chemical gradient could be caused by unevenly distribution of specific cell types. In the case of intestine, Paneth cells are restricted in the crypts and secrete Wnt3 and EGF for the maintenance of stem cell niche ${ }^{101}$. Along this line, by seeding different amounts of cells in defined location one could generate artificial chemical gradient cues in the later culture condition for organoid growth. In a recent study by Lancaster et al., the initial size of cell aggregates was shown to dictate the neuroectodermal formation of brain organoids ${ }^{102}$. In fact, the initial aggregate size was found to influence complex tissue organization and architecture or event cause failure in organoid development. An elegant method to control the seeding aggregates is to place defined number of cells in micropits that is microfabricated by soft lithography ${ }^{103,104}$ or stencil stamping into soft gels ${ }^{105}$. Soft lithography (Figure 2B) produces micropatterns by placing a mask between a UV light source and a photosensitive resin. After development, the arbitrary features on the photopolymer could be replicated by silicon-based organic polymers such as poly(dimethylsioxane) (PDMS), generating microscale pits or pillars on the surface. The PDMS replica can then be used directly as micropit arrays or to fabricate other hydrogel substrates by replica moulding. Stem cells that are seeded into these arrays are confined and when under centrifugal or gravity force, the confinement allows the formation of cell aggregates (Figure 2B). Additionally, different cells could be added into micropit arrays to increase component complexity and create interfaces among different cell types. This approach can also be conveniently scaled up to produce organoid arrays for several translational applications. In a recent example, PEG micropit arrays have been mass 
fabricated and directly anchored at the bottom of conventional multiwell plates for trapping stem cell aggregates at predefined focal plane ${ }^{106}$. In this way, large arrays of patient-derived colorectal cancer organoids were generated with high reproducibility and homogeneity for high-throughput anticancer drug screening. The combination with fast imaging and automated computations then enables high-content image-based phenotypic analyses to gain insights into drug-response mechanisms by real-time analysis of thousands of organoids.

Following the request to control cell-cell and tissue-tissue interface for adding another level of complexity to organoids, 3D bioprinting technologies (Figure $2 \mathrm{C}$ ) using biocompatible ink ${ }^{107}$ have been applied to the organoid filed. The bio-inks are normally a mixture of hydrogel and soluble nutrients to support the survival, proliferation and growth of cells or organoids. In comparison with homogenous co-culture method ${ }^{30}, 3 \mathrm{D}$ bioprinting allows different living cells/organoids to be embedded into various bio-inks and deposited by multiple ejectors in precise locations in an addictive layer-by-layer fashion. With this approach, cell masses containing multiple lineages and complicated structures could be obtained according to needs. For instance, the fusion of human cortical and thalamic organoids in vitro recapitulates the thalamocortical projections between thalamus and cortex ${ }^{108}$. This requires close position of these two types of organoids next to each ${ }^{109}$, which is, however, highly stochastic and labor-intensive. Precise spatial manipulation offered by 3D bioprinting should help to create robust, user-defined organoid-organoid interfaces and to decipher the principal mechanisms for tissue regeneration and interactions. In a recent work by Brassard and colleagues, mouse stomach corpus stem cells and ISCs were bioprinted inside 3D culture matrices to create centimeter long tubular tissues to mimic gastrointestinal tract ${ }^{110}$ for applications in drug discovery and regenerative medicine. Additionally, organoids that mimic liver, muscle, and blood vessels have been created by 3D bio-printing for toxicology testing ${ }^{111}$. The precise manipulation of stem cell niches then provides an interesting possibility for integrating multiple tissues to kick-off the formation of an multi-organoid system and for the study of their interactions and fusion in later remodeling ${ }^{112}$.

\section{Controlling mechanical cues in organoid systems}


As briefly mentioned above, external mechanical properties are known to affect stem cell fate and behaviors with a rivalry potency to biochemical signals. Indeed, stiffness alone can specify the differentiation of stem cells ${ }^{113}$ in 2D context. In the case of organoids, Capeling et al. ${ }^{114}$, have demonstrated that mechanical support alone is sufficient to foster human intestinal organoids survival and development. Using alginate as a minimal support, the researchers found human intestinal organoids differentiated indistinguishably from those grown inside Matrigel. Recent bioengineering efforts have focused on developing multiple approaches to provide inherent, instructive mechanical cues according to different developmental stages of organoids. This is elegantly exemplified by Gjorevski et al. ${ }^{82}$, who embedded ISCs in a mixture of mechanically static and dynamic PEG gels. The ratio between these two gels decided the degradability of the mixed matrix, allowing a control over the gel softening profile over time. The intestinal organoids inside the gel then experienced a decreasing mechanical elasticity. Their set of experiments interrogated the effects of external mechanical properties on intestinal organoid development and found that ISC expansion was enhanced by a high matrix stiffness while a soft matrix and laminin adhesion were optimal for ISC differentiation. Hence, this approach may mimic tissue evolution in responses to ever-shifting characteristics of native ECM. Similarly, in allyl sulfide hydrogels with photocleavable bonds, crypt-like structures formation in intestinal organoids was improved with matrix softening by photochemically induced degradation ${ }^{115}$. Additionally, the crypt-formation was accompanied with the differentiation of ISCs into Paneth cells. These studies underscore the interdependence between stem cell fates and material mechanical properties and such active matrices then allow eliciting defined ISC behavior patterns for intestinal organoid formation at certain time point or with light control. The latter case is particularly interesting as the photodegradable material can be easily combined with light-based patterning technologies ${ }^{116}$. However, degradable hydrogels might limit the long-term culture of organoids, as their supportive networks become unstable during irreversible degradation. Ideally, crosslinked networks of bio-hydrogels should be photoreversibly controlled via different wavelengths (or by other means) and as such, one can induce local/overall changes in the gel modulus at any time to modulate ISC behavior. Furthermore, many tissue developments in vivo are associated with stable mechanical strain. To investigate the effects of such strain, Poling and colleagues $^{117}$ recently incorporated a compressed nitinol spring into mesentery of mice, where human intestinal organoids were grafted. Under the stimulus of uniaxial strain, the 
transplanted organoids were found to have promoted intestinal maturation as well as nativelike transcription and morphology. Similarly, using in vitro stretching devices to actuate organoids also promote the growth, patterning and morphogenesis in human neural tube organoids derived from single hPSC ${ }^{118}$. These technologies would further improve our understanding of the role physical interactions via mechanical forces in regulating complex spatial organization in tissue.

\section{Regulating topographic cues for organoid growth}

Conventional organoids embedded in uniform 3D hydrogel without topographic features usually form a spherical architecture enclosing a lumen. Such an architecture exhibits a gap between in vitro and in vivo configuration, where native tissue often present highly regular shapes and organizations. For instance, small intestine epithelium lines the mucosa and organizes into highly regular crypt and villus structures, where cells of varied functions are found. Hence, 3D topographic cues of ECM may provide boundary conditions and guidance for the tissue architecture formation and integration. Indeed, when transferred from 2D substrates to 3D scaffolds, epithelia need to regulate its tissue contractility according to the geometry for the preservation of the tissue integrity ${ }^{119}$. Mammary epithelial cells may also take advantage of geometric cues to initiate morphogenesis processes, such as branching from epithelial tubules that are confined by the shape of hydrogel matrices is highly consistent with the patterned geometry ${ }^{105}$. Thus, it is necessary to fabricate 3D scaffolds with appropriate micro-structures to mimics physiological boundaries. A recent attempt in this direction was achieved by combining photopolymerizable hydrogels with high-resolution stereolithography to generate crypt and villus arrays to mimic the intestinal epithelium ${ }^{120}$ (Figure 2C). This 3D scaffold could support the growth of Caco-2 cells for 3 weeks, leading to a differentiated epithelial layer. Another example also used photolithography and molding to fabricate similar crypt and villus micropatterns in collagen substrates ${ }^{121}$. Using these 3D scaffolds and under a conventional culture condition for intestinal organoid expansion, ISCS from human intestinal organoids formed a 3D self-renewing epithelium. In addition, by combining the topography with gradients of morphogens, the ISCs were guided into in vivolike configuration recapitulating cell-specific compartments and distribution with directional cell migration along the crypt-villus axis. The same group of researchers also used a similar 
approach to reproduce in vitro crypt structures for human colonic epithelium, mimicking multiple aspects of colonic tissue ${ }^{122}$. These studies provide means to control topographic cues and demonstrate their importance for organoid development. Of note, such an ex vivo models offer an open apical part of the tissue, thus allowing a broad range of physiological relevant experiments, such as epithelial defense, metabolism, and studies of drugs, microbiota and toxins in intestinal context.

\section{Spatiotemporal control of diffusive biochemical signals}

During organogenesis, gradients of biochemical signals including morphogens and growth factors play an important role in triggering symmetry breaking, polarization, and pattern formation in the tissue. However, conventional organoids are flooded with homogeneous medium without any spatiotemporal modulation of these cues, resulting in significant differences between in vivo and in vitro tissue development and organization. To overcome this drawback, microfluidic systems have been employed to recreate the biochemical gradients for organoid growth and organization. For instance, colonic organoids or individual stem cells had been placed in a microengineered gradient channel, where linear gradients of Wnt3a and R-spondin1 were generated by diffusion ${ }^{123}$. Such an exposure caused higher degree of polarization in colonoids in compared to those cultured without a gradient. It is also noticeable that stem cells were polarized more in the form of single cells than those within colonoid fragments, suggesting that the cell clusters may already have other intrinsic prepatterns that counteract the influences of external stimuli. Besides microfluidics, biochemical gradient can also be created by tethering biomolecules, whose active sites are masked with a photo-degradable moiety, into a hydrogel matrix, rendering photo-responsive release ${ }^{124}$. This method is also compatible with photo-patterning technologies, enabling precise creation of localized biochemical patterns within a matrix with light. In a work using this photodegradable tethering approach ${ }^{125}$, a peptide (sequence Arg-Gly-Asp-Ser, RGDS) coupled to a PEG hydrogel via photolabile moieties was cleaved from the network upon selective irradiation. The release of the peptide RGDS then led embedded human mesenchymal stem cells down the chondrogenic pathway. Similarly, nanoparticles or degradable vehicles can be loaded with growth factors and tethered to cells or the networks of hydrogel to mimic autocrine, 
paracrine, and juxtacrine signals ${ }^{68}$. Further efforts are still required to fully realize the potentials of these techniques in the field of organoid.

\section{Monitoring organoid components in vitro}

The aforementioned bioengineering approaches offer means to control multiple cues to guide organoid development. It is also necessary to monitor components in the organoid systems, such as cell-cell/matrix interactions, intrinsic genetic information, local nutrient transport and oxygen levels. Sensors and detectors could be embedded in in vitro set-ups for in situ read-out of the influence of cell-mediated signaling. For instance, micromachined silicon substrates could manipulate adherent cells with micrometer-scale precision and study their interactions ${ }^{126}$. To measure and manipulate organoid mechanics, multiple methods and tools have been developed. These include 3D traction force microscopy ${ }^{127}$, elastic soft droplet insert $^{128}$, laser ablation ${ }^{129}$ and fluorescence resonance energy transfer tension sensors ${ }^{130}$. Applying these techniques to organoid systems to probe 3D organoid mechanics have resulted in some successes ${ }^{131}$. Moreover, computational frameworks and simulation models can be used to predict and support mechanical measurement of 3D organoid dynamics ${ }^{132,133}$. In a recent work, Karzbrun and colleagues modeled the role of mechanical forces in guiding surface wrinkles in human brain organoids trapped in a microfabricated compartment ${ }^{134}$. There are also other analytical tools that are able to be included in microfabricated platforms for organoid studies, such as micro-electrode arrays ${ }^{135}$, mass cytometry imaging ${ }^{136}$ and microflourimetry ${ }^{19}$. Besides these, the recent advances in transcriptomics have offered new opportunities to unravel in-depth organoid genetic information. RNA sequencing of kidney organoids at different time points have revealed transcriptional profiles that indicate early cultured organoids highly matching the fetal gonad ${ }^{137}$. Transcriptomic analysis can also serve as guidelines to fine tune 3D extracellular environment for the generation of kidney organoids showing congruence with second-trimester human fetal kidneys ${ }^{138}$. Taken all together, combining multiple analytical tools to organoid systems in spatiotemporal manners would be capable to provide biochemical, tissue mechanical and genome-wide signatures for different cells within organoids at different developmental stages. 


\section{Bioengineered integration of multiple components for organoid assembly}

The presence of multiple parameters, such as the biochemical and physical properties of the matrix, soluble morphogens, mechanical strains, as well as topographic cues, in organogenesis ${ }^{124}$ suggests that a systematic integration of these variables is essential to achieve customized, bioengineered in vitro organoid systems. At present, one promising approach in this direction is microfluidic organs-on-a-chip system established through synergistic engineering ${ }^{139}$. Such platforms could integrate numerous functional components that offer control over cell culture, shear stress, mechanical strain, spatial confinement, topographic patterns, biochemical gradients, electrical stimulation, and sensors for signal readouts. As such, there is a trend in converging the versatility of organs-on-a-chip systems and organoid-based tissue engineering to bestow artificial guidance to current organoid technologies. To achieve this, these functional modules must be systematically associated into one chip to tune each factor individually and synchronously without interfering with the autonomous stem cell self-assembly capability.

In a previous example, microfluidic chips for mimicking human intestine, named gut-on-achip $^{140-142}$, were produced to provide controllable microenvironment for ISC culture (Figure 3). In such a system, a flexible, porous membrane was connected to two vacuum chambers and separated the cell culture channel into top and bottom parts. The membrane was functionalized with ECM to support cell survival and expansion. Cyclic mechanical strains mimicking peristalsis caused by the extension and retraction of the membrane, were transmitted via the deformation of the two hollow side chambers upon suction and biochemical gradient across the porous membrane is generated by perfusing different culture media into the top and bottom culture channels. Additionally, 3D scaffolds that mimic the crypt-villus axis could be mounted on the supportive membrane to provide physical boundaries for the preservation of tissue architectures. Primary intestinal epithelial cells from biopsy-derived organoids were then introduced in the top surface of the membrane, forming a parenchymal channel, whereas microvascular endothelial and immune cells were cocultured on the opposite side, representing a vascular channel ${ }^{141,142}$. Moreover, human intestinal commensal microbiota could be co-cultured in the upper channel, in contact with 
living intestinal epithelia and under physiological shear flow ${ }^{143}$. Hence, microfluidics-based integration offers unparalleled control over individual biochemical, biophysical, mechanical, cellular and bacterial factors in an artificial intestine model. This can allow researchers to explore molecular, cellular and tissue mechanisms and gain insights into intestinal physiology and pathology. Besides intestinal model, a biomimetic lung-on-chip model has been developed via similar principles to study multiple lung functions ${ }^{144}$. Hence, one can also easily incorporate cells from multiple species, i.e., human and animal cells, in a same chip, to determine the conserved tissue development and functional mechanisms. The same integration principles are applicable to the establishment of other organ-on-a-chip and cancer-on-a-chip systems ${ }^{145}$. Such platforms should then have important utilities in pharmaceutical screening and disease modeling in that they have potentials to significantly reduce cost and can avoid the ethical issues with animal models. In short, novel organ-on-achips offer new capacities to control numerous biochemical and biophysical parameters and to analyze their contributions to human physiology and pathophysiology one-at-a-time.

\section{Concluding remarks}

In the past decades, we have witnessed numerous breakthroughs in the field of organoid technologies. A series of organ-specific organoids have been developed and the advancements in organoid-based tissue engineering have significantly improved our understandings in organogenesis and therapy strategies. However, the further utility of organoid to reproduce functional organs in vitro is currently hindered by the highly variable, unmatured differentiated, and poorly organized self-assembly systems. To overcome these challenges inherent in autonomous organoid systems, a few impressive steps towards the convergence of bioengineering approaches and organoid systems have been made. Bioengineered tools derived from microfabrication, genome editing, designer biomaterials, and other advanced microtechnologies have been successfully implemented for the manipulation of cellular microenvironment and stem cells themselves to direct cell-substrate and cell-cell interactions. This rich toolbox has particular contributed to reveal many morphogenetic rules and the independent effects of physiological environmental parameters 
that are largely intertwined in vivo in modulating stem cell and organoid development. While it is not without challenges to integrate these tools in an organoid system without tampering with inherent biological qualities and capabilities, we envision that the continuous expansion of this bioengineering library will further empower researchers to develop novel biomimetic organ-on-chips systems to advance our knowledge in many fundamental biology and to transform regenerative therapies. 


\section{Abbreviations:}

2D: two-dimensional

3D: three-dimensional

ECM: extracellular matrix

ASC: adult stem cell

iPSC: induced pluripotent stem cell

ESC: embryonic stem cell

ISC: intestinal stem cell

PEG: poly(ethylene glycol)

PAA: polyacrylamine

PDMS: poly(dimethylsioxane)

RGDS: peptide sequence Arg-Gly-Asp-Ser

MMPs: matrix metalloproteinases

VEGF: vascular endothelial growth-factor

Authorship: J.S., B.M. and W.X. discussed the content of the review. J.S., B.M. and W.X. wrote manuscript. J.S. and W.X. made the figures. J.S. and W.X. reviewed, edited and revised the manuscript.

Acknowledgments: We sincerely thank Delphine Delacour for very helpful discussion and suggestions. We thank all members of the cell adhesion and mechanics Lab for their help and support.

Funding: This work was supported by the Marie Skłodowska-Curie Actions (W.X., Individual Fellowship, Project: 846449), funding from the People Programme (Marie Curie Actions) of the European Union's Seventh Framework Programme (FP7/2007-2013) under REA grant agreement no. PCOFUND-GA-2013-609102, through the PRESTIGE programme coordinated by Campus France, the University of Paris (J.S. and B.M.), and grants from the Groupama Foundation - Research Prize for Rare Diseases 2017 (W.X. and J.S.), the LabEx “Who Am I?" \#ANR-11-LABX-0071 and the Universite de Paris IdEx \#ANR-18-IDEX-0001 funded by the French Government through its "Investments for the Future" program (W.X. and J.S.), the 
Human Frontier Science Program (RGP0038/2018) (W.X. and J.S.), and ANR Mecanoadipo (B.M.).

Conflict of Interest Disclosure: The authors declare that no competing interests exist.

\section{References}

1 Shanks, N., Greek, R. \& Greek, J. Are animal models predictive for humans? Philosophy, Ethics, and Humanities in Medicine 4, 2 (2009).

2 Marx, V. Cell-line authentication demystified. Nature Methods 11, 483-488 (2014).

3 Zhou, J., Su, J., Fu, X., Zheng, L. \& Yin, Z. Microfluidic device for primary tumor spheroid isolation. Exp Hematol Oncol 6, 22-22 (2017).

4 Wang, F. et al. Reciprocal interactions between beta1-integrin and epidermal growth factor receptor in three-dimensional basement membrane breast cultures: a different perspective in epithelial biology. Proc Natl Acad Sci U S A 95, 14821-14826 (1998).

5 McKee, C. \& Chaudhry, G. R. Advances and challenges in stem cell culture. Colloids and surfaces. B, Biointerfaces 159, 62-77 (2017).

6 Wicha, M. S., Liotta, L. A., Vonderhaar, B. K. \& Kidwell, W. R. Effects of inhibition of basement membrane collagen deposition on rat mammary gland development. Developmental Biology 80, 253-266 (1980).

7 Roskelley, C. D. \& Bissell, M. J. The dominance of the microenvironment in breast and ovarian cancer. Seminars in cancer biology 12, 97-104 (2002).

8 Emerman, J. T. \& Pitelka, D. R. Maintenance and induction of morphological differentiation in dissociated mammary epithelium on floating collagen membranes. In Vitro 13, 316-328 (1977).

9 Petersen, O. W., Rønnov-Jessen, L., Howlett, A. R. \& Bissell, M. J. Interaction with basement membrane serves to rapidly distinguish growth and differentiation pattern of normal and malignant human breast epithelial cells. Proc. Natl. Acad. Sci. U.S.A 89, 9064-9068 (1992).

10 Debnath, J. \& Brugge, J. S. Modelling glandular epithelial cancers in three-dimensional cultures. Nature reviews. Cancer 5, 675-688 (2005).

11 Debnath, J., Muthuswamy, S. K. \& Brugge, J. S. Morphogenesis and oncogenesis of MCF-10A mammary epithelial acini grown in three-dimensional basement membrane cultures. Methods (San Diego, Calif.) 30, 256-268 (2003).

12 Muthuswamy, S. K., Li, D., Lelievre, S., Bissell, M. J. \& Brugge, J. S. ErbB2, but not ErbB1, reinitiates proliferation and induces luminal repopulation in epithelial acini. Nat. Cell Biol. 3, 785-792 (2001).

13 Debnath, J., Walker, S. J. \& Brugge, J. S. Akt activation disrupts mammary acinar architecture and enhances proliferation in an mTOR-dependent manner. J Cell Biol 163, 315-326 (2003).

14 Eiraku, M. et al. Self-Organized Formation of Polarized Cortical Tissues from ESCs and Its Active Manipulation by Extrinsic Signals. Cell Stem Cell 3, 519-532 (2008).

15 Sato, T. et al. Single Lgr5 stem cells build crypt-villus structures in vitro without a mesenchymal niche. Nature 459, 262-265 (2009). 
16 Lancaster, M. A. \& Knoblich, J. A. Organogenesis in a dish: Modeling development and disease using organoid technologies. Science 345, 1247125 (2014).

17 Jabs, J. et al. Screening drug effects in patient-derived cancer cells links organoid responses to genome alterations. Molecular systems biology 13, 955 (2017).

18 Murphy, W. L., McDevitt, T. C. \& Engler, A. J. Materials as stem cell regulators. Nature materials 13, 547-557 (2014).

19 Yin, X. et al. Engineering Stem Cell Organoids. Cell Stem Cell 18, 25-38 (2016).

20 Brassard, J. A. \& Lutolf, M. P. Engineering Stem Cell Self-organization to Build Better Organoids. Cell Stem Cell 24, 860-876 (2019).

21 Huch, M. et al. Unlimited in vitro expansion of adult bi-potent pancreas progenitors through the Lgr5/R-spondin axis. The EMBO Journal 32, 2708-2721 (2013).

22 Karthaus, W. R. et al. Identification of multipotent luminal progenitor cells in human prostate organoid cultures. Cell 159, 163-175 (2014).

23 Barker, N. et al. Lgr5(+ve) stem cells drive self-renewal in the stomach and build longlived gastric units in vitro. Cell Stem Cell 6, 25-36 (2010).

24 Spence, J. R. et al. Directed differentiation of human pluripotent stem cells into intestinal tissue in vitro. Nature 470, 105-109 (2011).

25 McCracken, K. W. et al. Modelling human development and disease in pluripotent stem-cell-derived gastric organoids. Nature 516, 400-404 (2014).

26 Dye, B. R. et al. In vitro generation of human pluripotent stem cell derived lung organoids. elife 4, e05098 (2015).

27 Wells, J. M. \& Spence, J. R. How to make an intestine. Development 141, 752-760 (2014).

28 Lancaster, M. A. et al. Cerebral organoids model human brain development and microcephaly. Nature 501, 373-379 (2013).

29 Takasato, M. et al. Directing human embryonic stem cell differentiation towards a renal lineage generates a self-organizing kidney. Nat. Cell Biol. 16, 118-126 (2014).

30 Takebe, T. et al. Vascularized and functional human liver from an iPSC-derived organ bud transplant. Nature 499, 481-484 (2013).

31 Dye, B. R. et al. In vitro generation of human pluripotent stem cell derived lung organoids. Elife 4 (2015).

32 Wimmer, R. A. et al. Human blood vessel organoids as a model of diabetic vasculopathy. Nature 565, 505-510 (2019).

33 Eiraku, M. et al. Self-organizing optic-cup morphogenesis in three-dimensional culture. Nature 472, 51-56 (2011).

34 Taguchi, A. et al. Redefining the In Vivo Origin of Metanephric Nephron Progenitors Enables Generation of Complex Kidney Structures from Pluripotent Stem Cells. Cell Stem Cell 14, 53-67 (2014).

35 Taguchi, A. \& Nishinakamura, R. Higher-Order Kidney Organogenesis from Pluripotent Stem Cells. Cell Stem Cell 21, 730-746.e736 (2017).

36 Noguchi, T.-a. K. et al. Generation of stomach tissue from mouse embryonic stem cells. Nat. Cell Biol. 17, 984-993 (2015).

37 Sato, T. \& Clevers, H. Growing self-organizing mini-guts from a single intestinal stem cell: mechanism and applications. Science 340, 1190-1194 (2013).

38 Sasai, Y. Cytosystems dynamics in self-organization of tissue architecture. Nature 493, 318-326 (2013). 
39 Sato, T. et al. Paneth cells constitute the niche for Lgr5 stem cells in intestinal crypts. Nature 469, 415-418 (2011).

40 Cederquist, G. Y. et al. Specification of positional identity in forebrain organoids. Nat. Biotechnol. 37, 436-444 (2019).

41 Sato, T. et al. Long-term Expansion of Epithelial Organoids From Human Colon, Adenoma, Adenocarcinoma, and Barrett's Epithelium. Gastroenterology 141, 17621772 (2011).

42 Schwank, G. et al. Functional Repair of CFTR by CRISPR/Cas9 in Intestinal Stem Cell Organoids of Cystic Fibrosis Patients. Cell Stem Cell 13, 653-658 (2013).

43 Bartfeld, S. et al. In Vitro Expansion of Human Gastric Epithelial Stem Cells and Their Responses to Bacterial Infection. Gastroenterology 148, 126-136.e126 (2015).

44 Broutier, L. et al. Human primary liver cancer-derived organoid cultures for disease modeling and drug screening. Nature Medicine 23, 1424-1435 (2017).

45 Fujii, M. \& Sato, T. Somatic cell-derived organoids as prototypes of human epithelial tissues and diseases. Nature Materials 20, 156-169 (2021).

46 Coll, M. et al. Generation of Hepatic Stellate Cells from Human Pluripotent Stem Cells Enables In Vitro Modeling of Liver Fibrosis. Cell Stem Cell 23, 101-113.e107 (2018).

47 Yakeishi, Y., Mori, M. \& Enjoji, M. Distribution of beta-human chorionic gonadotropinpositive cells in noncancerous gastric mucosa and in malignant gastric tumors. Cancer 66, 695-701 (1990).

48 Grassi, L. et al. Organoids as a new model for improving regenerative medicine and cancer personalized therapy in renal diseases. Cell Death \& Disease 10, 201 (2019).

49 Clevers, H. Modeling Development and Disease with Organoids. Cell 165, 1586-1597 (2016).

50 Okamoto, R. et al. Organoid-based regenerative medicine for inflammatory bowel disease. Regenerative Therapy 13, 1-6 (2020).

51 Li, M. \& Izpisua Belmonte, J. C. Organoids - Preclinical Models of Human Disease. New England Journal of Medicine 380, 569-579 (2019).

52 Astashkina, A., Mann, B. \& Grainger, D. W. A critical evaluation of in vitro cell culture models for high-throughput drug screening and toxicity. Pharmacology \& Therapeutics 134, 82-106 (2012).

53 Co, J. Y. et al. Controlling Epithelial Polarity: A Human Enteroid Model for HostPathogen Interactions. Cell Rep 26, 2509-2520.e2504 (2019).

54 Workman, M. J. et al. Engineered human pluripotent-stem-cell-derived intestinal tissues with a functional enteric nervous system. Nature Medicine 23, 49-59 (2017).

55 Dijkstra, K. K. et al. Generation of Tumor-Reactive T Cells by Co-culture of Peripheral Blood Lymphocytes and Tumor Organoids. Cell 174, 1586-1598.e1512 (2018).

56 Yui, S. et al. YAP/TAZ-Dependent Reprogramming of Colonic Epithelium Links ECM Remodeling to Tissue Regeneration. Cell Stem Cell 22, 35-49.e37 (2018).

57 Velazquez, J. J., Su, E., Cahan, P. \& Ebrahimkhani, M. R. Programming Morphogenesis through Systems and Synthetic Biology. Trends in Biotechnology 36, 415-429 (2018).

58 Guye, P. et al. Genetically engineering self-organization of human pluripotent stem cells into a liver bud-like tissue using Gata6. Nat. Commun. 7, 10243 (2016).

59 Schwank, G. \& Clevers, H. CRISPR/Cas9-Mediated Genome Editing of Mouse Small Intestinal Organoids. Methods in molecular biology (Clifton, N.J.) 1422, 3-11 (2016).

60 Koo, B.-K. et al. Controlled gene expression in primary Lgr5 organoid cultures. Nature Methods 9, 81-83 (2012). 
61 Fujii, M., Matano, M., Nanki, K. \& Sato, T. Efficient genetic engineering of human intestinal organoids using electroporation. Nat. Protoc. 10, 1474-1485 (2015).

62 Drost, J. et al. Sequential cancer mutations in cultured human intestinal stem cells. Nature 521, 43-47 (2015).

63 Matano, M. et al. Modeling colorectal cancer using CRISPR-Cas9-mediated engineering of human intestinal organoids. Nature Medicine 21, 256-262 (2015).

64 Todhunter, M. E. et al. Programmed synthesis of three-dimensional tissues. Nature Methods 12, 975-981 (2015).

65 Gartner, Z. J. \& Bertozzi, C. R. Programmed assembly of 3-dimensional microtissues with defined cellular connectivity. Proc. Natl. Acad. Sci. U.S.A 106, 4606-4610 (2009).

66 O'Brien, P. J., Luo, W., Rogozhnikov, D., Chen, J. \& Yousaf, M. N. Spheroid and Tissue Assembly via Click Chemistry in Microfluidic Flow. Bioconjugate Chemistry 26, 19391949 (2015).

67 Zhao, W. et al. Mimicking the inflammatory cell adhesion cascade by nucleic acid aptamer programmed cell-cell interactions. The FASEB Journal 25, 3045-3056 (2011).

68 Stephan, M. T. \& Irvine, D. J. Enhancing cell therapies from the outside in: Cell surface engineering using synthetic nanomaterials. Nano Today 6, 309-325 (2011).

69 Suga, H. et al. Self-formation of functional adenohypophysis in three-dimensional culture. Nature 480, 57-62 (2011).

70 Daley, W. P., Peters, S. B. \& Larsen, M. Extracellular matrix dynamics in development and regenerative medicine. J. Cell Sci. 121, 255-264 (2008).

71 Hughes, C. S., Postovit, L. M. \& Lajoie, G. A. Matrigel: a complex protein mixture required for optimal growth of cell culture. Proteomics 10, 1886-1890 (2010).

72 Hussey, G. S., Cramer, M. C. \& Badylak, S. F. Extracellular Matrix Bioscaffolds for Building Gastrointestinal Tissue. CMGH Cell. Mol. Gastroenterol. Hepatol. 5, 1-13 (2017).

73 Badylak, S. F. Xenogeneic extracellular matrix as a scaffold for tissue reconstruction. Transplant Immunology 12, 367-377 (2004).

74 Badylak, S. F., Freytes, D. O. \& Gilbert, T. W. Extracellular matrix as a biological scaffold material: Structure and function. Acta Biomaterialia 5, 1-13 (2009).

75 Gilbert, T. W., Stolz, D. B., Biancaniello, F., Simmons-Byrd, A. \& Badylak, S. F. Production and characterization of ECM powder: implications for tissue engineering applications. Biomaterials 26, 1431-1435 (2005).

76 Song, J. J. \& Ott, H. C. Organ engineering based on decellularized matrix scaffolds. Trends in molecular medicine 17, 424-432 (2011).

77 Schweinlin, M. et al. Development of an Advanced Primary Human In Vitro Model of the Small Intestine. Tissue engineering. Part C, Methods 22, 873-883 (2016).

78 Tong, Z. et al. Towards a defined ECM and small molecule based monolayer culture system for the expansion of mouse and human intestinal stem cells. Biomaterials 154, 60-73 (2018).

79 Xi, W., Saw, T. B., Delacour, D., Lim, C. T. \& Ladoux, B. Material approaches to active tissue mechanics. Nat. Rev. Mater. 4, 23-44 (2019).

80 Lu, Y., Aimetti, A. A., Langer, R. \& Gu, Z. Bioresponsive materials. Nat. Rev. Mater. 2, 16075 (2016).

81 Rosales, A. M. \& Anseth, K. S. The design of reversible hydrogels to capture extracellular matrix dynamics. Nat. Rev. Mater. 1, 15012 (2016). 
82 Gjorevski, N. et al. Designer matrices for intestinal stem cell and organoid culture. Nature 539, 560-564 (2016).

83 Cruz-Acuña, R. et al. Synthetic hydrogels for human intestinal organoid generation and colonic wound repair. Nat. Cell Biol. 19, 1326-1335 (2017).

84 Ranga, A. et al. 3D niche microarrays for systems-level analyses of cell fate. Nat. Commun. 5, 4324 (2014).

85 Gobaa, S. et al. Artificial niche microarrays for probing single stem cell fate in high throughput. Nature Methods 8, 949-955 (2011).

86 Yim, E. K. F. et al. Nanopattern-induced changes in morphology and motility of smooth muscle cells. Biomaterials 26, 5405-5413 (2005).

87 McMurray, R. J. et al. Nanoscale surfaces for the long-term maintenance of mesenchymal stem cell phenotype and multipotency. Nature Materials 10, 637-644 (2011).

88 Lei, Y. \& Schaffer, D. V. A fully defined and scalable 3D culture system for human pluripotent stem cell expansion and differentiation. Proc. Natl. Acad. Sci. U.S.A 110, E5039-E5048 (2013).

89 Swioklo, S., Ding, P., Pacek, A. W. \& Connon, C. J. Process parameters for the highscale production of alginate-encapsulated stem cells for storage and distribution throughout the cell therapy supply chain. Process Biochemistry 59, 289-296 (2017).

90 Horiguchi, I. \& Sakai, Y. Alginate Encapsulation of Pluripotent Stem Cells Using a Coaxial Nozzle. J Vis Exp, e52835-e52835 (2015).

91 Fattahi, P. et al. Core-shell hydrogel microcapsules enable formation of human pluripotent stem cell spheroids and their cultivation in a stirred bioreactor. Sci. Rep. 11, 7177 (2021).

92 Blondel, D. \& Lutolf, M. P. Bioinspired Hydrogels for 3D Organoid Culture. Chimia 73, 81-85 (2019).

93 Kuhlman, W., Taniguchi, I., Griffith, L. G. \& Mayes, A. M. Interplay Between PEO Tether Length and Ligand Spacing Governs Cell Spreading on RGD-Modified PMMA-g-PEO Comb Copolymers. Biomacromolecules 8, 3206-3213 (2007).

94 Fonseca, K. B., Granja, P. \& Barrias, C. Engineering proteolytically-degradable artificial extracellular matrices. Progress in Polymer Science 39, 2010-2029 (2014).

95 Huang, G. et al. Functional and Biomimetic Materials for Engineering of the ThreeDimensional Cell Microenvironment. Chem. Rev. 117, 12764-12850 (2017).

96 Raeber, G. P., Lutolf, M. P. \& Hubbell, J. A. Molecularly Engineered PEG Hydrogels: A Novel Model System for Proteolytically Mediated Cell Migration. Biophys. J. 89, 13741388 (2005).

97 Baker, B. M. et al. Cell-mediated fibre recruitment drives extracellular matrix mechanosensing in engineered fibrillar microenvironments. Nature Materials 14, 1262-1268 (2015).

98 Guvendiren, M. \& Burdick, J. A. Stiffening hydrogels to probe short- and long-term cellular responses to dynamic mechanics. Nat. Commun. 3 (2012).

99 Ondeck, M. G. et al. Dynamically stiffened matrix promotes malignant transformation of mammary epithelial cells via collective mechanical signaling. Proc. Natl. Acad. Sci. U.S.A 116, 3502-3507 (2019).

100 Phelps, E. A., Landázuri, N., Thulé, P. M., Taylor, W. R. \& García, A. J. Bioartificial matrices for therapeutic vascularization. Proc. Natl. Acad. Sci. U.S.A 107, 3323-3328 (2010). 
101 Wang, Y. et al. Bioengineered Systems and Designer Matrices That Recapitulate the Intestinal Stem Cell Niche. CMGH Cell. Mol. Gastroenterol. Hepatol. 5, 440-453.e441 (2018).

102 Lancaster, M. A. et al. Guided self-organization and cortical plate formation in human brain organoids. Nat. Biotechnol. 35, 659-666 (2017).

103 Shin, H.-S., Hong, H. J., Koh, W.-G. \& Lim, J.-Y. Organotypic 3D Culture in Nanoscaffold Microwells Supports Salivary Gland Stem-Cell-Based Organization. ACS Biomaterials Science \& Engineering 4, 4311-4320 (2018).

104 Czerniecki, S. M. et al. High-Throughput Screening Enhances Kidney Organoid Differentiation from Human Pluripotent Stem Cells and Enables Automated Multidimensional Phenotyping. Cell Stem Cell 22, 929-940.e924 (2018).

105 Nelson, C. M., VanDuijn, M. M., Inman, J. L., Fletcher, D. A. \& Bissell, M. J. Tissue geometry determines sites of mammary branching morphogenesis in organotypic cultures. Science 314, 298-300 (2006).

106 Brandenberg, N. et al. High-throughput automated organoid culture via stem-cell aggregation in microcavity arrays. Nature Biomedical Engineering 4, 863-874 (2020).

107 Murphy, S. V. \& Atala, A. 3D bioprinting of tissues and organs. Nat. Biotechnol. 32, 773-785 (2014).

108 Xiang, Y. et al. hESC-Derived Thalamic Organoids Form Reciprocal Projections When Fused with Cortical Organoids. Cell Stem Cell 24, 487-497.e487 (2019).

109 Xiang, Y. et al. Fusion of Regionally Specified hPSC-Derived Organoids Models Human Brain Development and Interneuron Migration. Cell Stem Cell 21, 383-398.e387 (2017).

110 Brassard, J. A., Nikolaev, M., Hübscher, T., Hofer, M. \& Lutolf, M. P. Recapitulating macro-scale tissue self-organization through organoid bioprinting. Nature Materials 20, 22-29 (2021).

111 Kang, H.-W., Atala, A. \& Yoo, J. J. in Essentials of 3D Biofabrication and Translation (eds Anthony Atala \& James J. Yoo) 179-186 (Academic Press, 2015).

112 Guven, S. et al. Multiscale assembly for tissue engineering and regenerative medicine. Trends in Biotechnology 33, 269-279 (2015).

113 Engler, A. J., Sen, S., Sweeney, H. L. \& Discher, D. E. Matrix Elasticity Directs Stem Cell Lineage Specification. Cell 126, 677-689 (2006).

114 Capeling, M. M. et al. Nonadhesive Alginate Hydrogels Support Growth of Pluripotent Stem Cell-Derived Intestinal Organoids. Stem Cell Reports 12, 381-394 (2019).

115 Hushka, E. A., Yavitt, F. M., Brown, T. E., Dempsey, P. J. \& Anseth, K. S. Relaxation of Extracellular Matrix Forces Directs Crypt Formation and Architecture in Intestinal Organoids. Advanced Healthcare Materials 9, 1901214 (2020).

116 Strale, P.-O. et al. Multiprotein Printing by Light-Induced Molecular Adsorption. Adv. Mater. 28, 2024-2029 (2016).

117 Poling, H. M. et al. Mechanically induced development and maturation of human intestinal organoids in vivo. Nature Biomedical Engineering 2, 429-442 (2018).

118 Abdel Fattah, A. R. et al. Actuation enhances patterning in human neural tube organoids. Nat. Commun. 12, 3192 (2021).

119 Salomon, J. et al. Contractile forces at tricellular contacts modulate epithelial organization and monolayer integrity. Nat. Commun. 8, 13998 (2017).

120 Creff, J. et al. Fabrication of 3D scaffolds reproducing intestinal epithelium topography by high-resolution 3D stereolithography. Biomaterials 221, 119404 (2019). 
121 Wang, Y. et al. A microengineered collagen scaffold for generating a polarized cryptvillus architecture of human small intestinal epithelium. Biomaterials 128, 44-55 (2017).

122 Wang, Y. et al. Formation of Human Colonic Crypt Array by Application of Chemical Gradients Across a Shaped Epithelial Monolayer. CMGH Cell. Mol. Gastroenterol. Hepatol. 5, 113-130 (2018).

123 Attayek, P. J. et al. In Vitro Polarization of Colonoids to Create an Intestinal Stem Cell Compartment. PLOS ONE 11, e0153795 (2016).

124 Gjorevski, N., Ranga, A. \& Lutolf, M. P. Bioengineering approaches to guide stem cellbased organogenesis. Development 141, 1794-1804 (2014).

125 Kloxin, A. M., Kasko, A. M., Salinas, C. N. \& Anseth, K. S. Photodegradable Hydrogels for Dynamic Tuning of Physical and Chemical Properties. Science 324, 59-63 (2009).

126 Hui, E. E. \& Bhatia, S. N. Micromechanical control of cell-cell interactions. Proc. Natl. Acad. Sci. U.S.A 104, 5722-5726 (2007).

127 Legant, W. R. et al. Measurement of mechanical tractions exerted by cells in threedimensional matrices. Nature Methods 7, 969-971 (2010).

128 Campàs, O. et al. Quantifying cell-generated mechanical forces within living embryonic tissues. Nature Methods 11, 183 (2014).

129 Pérez-González, C. et al. Mechanical compartmentalization of the intestinal organoid enables crypt folding and collective cell migration. Nat. Cell Biol. 23, 745-757 (2021).

130 Grashoff, C. et al. Measuring mechanical tension across vinculin reveals regulation of focal adhesion dynamics. Nature 466, 263-266 (2010).

131 Garreta, E. et al. Rethinking organoid technology through bioengineering. Nature Materials 20, 145-155 (2021).

132 Etournay, R. et al. TissueMiner: A multiscale analysis toolkit to quantify how cellular processes create tissue dynamics. elife 5, e14334 (2016).

133 Latorre, E. et al. Active superelasticity in three-dimensional epithelia of controlled shape. Nature 563, 203-208 (2018).

134 Karzbrun, E., Kshirsagar, A., Cohen, S. R., Hanna, J. H. \& Reiner, O. Human brain organoids on a chip reveal the physics of folding. Nat. Phys. 14, 515-522 (2018).

135 Passaro, A. P. \& Stice, S. L. Electrophysiological Analysis of Brain Organoids: Current Approaches and Advancements. Frontiers in Neuroscience 14 (2021).

136 Bodenmiller, B. Multiplexed Epitope-Based Tissue Imaging for Discovery and Healthcare Applications. Cell Systems 2, 225-238 (2016).

137 Takasato, M. et al. Kidney organoids from human iPS cells contain multiple lineages and model human nephrogenesis. Nature 526, 564-568 (2015).

138 Garreta, E. et al. Fine tuning the extracellular environment accelerates the derivation of kidney organoids from human pluripotent stem cells. Nature Materials 18, 397-405 (2019).

139 Zhang, B., Korolj, A., Lai, B. F. L. \& Radisic, M. Advances in organ-on-a-chip engineering. Nat. Rev. Mater. 3, 257-278 (2018).

140 Shin, W., Hinojosa, C. D., Ingber, D. E. \& Kim, H. J. Human Intestinal Morphogenesis Controlled by Transepithelial Morphogen Gradient and Flow-Dependent Physical Cues in a Microengineered Gut-on-a-Chip. iScience 15, 391-406 (2019).

141 Bein, A. et al. Microfluidic Organ-on-a-Chip Models of Human Intestine. CMGH Cell. Mol. Gastroenterol. Hepatol. 5, 659-668 (2018). 
142 Kasendra, M. et al. Development of a primary human Small Intestine-on-a-Chip using biopsy-derived organoids. Sci. Rep. 8, 2871 (2018).

143 Kim, H. J., Li, H., Collins, J. J. \& Ingber, D. E. Contributions of microbiome and mechanical deformation to intestinal bacterial overgrowth and inflammation in a human gut-on-a-chip. Proc. Natl. Acad. Sci. U.S.A 113, E7-E15 (2016).

144 Huh, D. et al. Reconstituting Organ-Level Lung Functions on a Chip. Science 328, $1662-$ 1668 (2010).

145 Bhatia, S. N. \& Ingber, D. E. Microfluidic organs-on-chips. Nat Biotech 32, 760-772 (2014). 


\section{Figures}

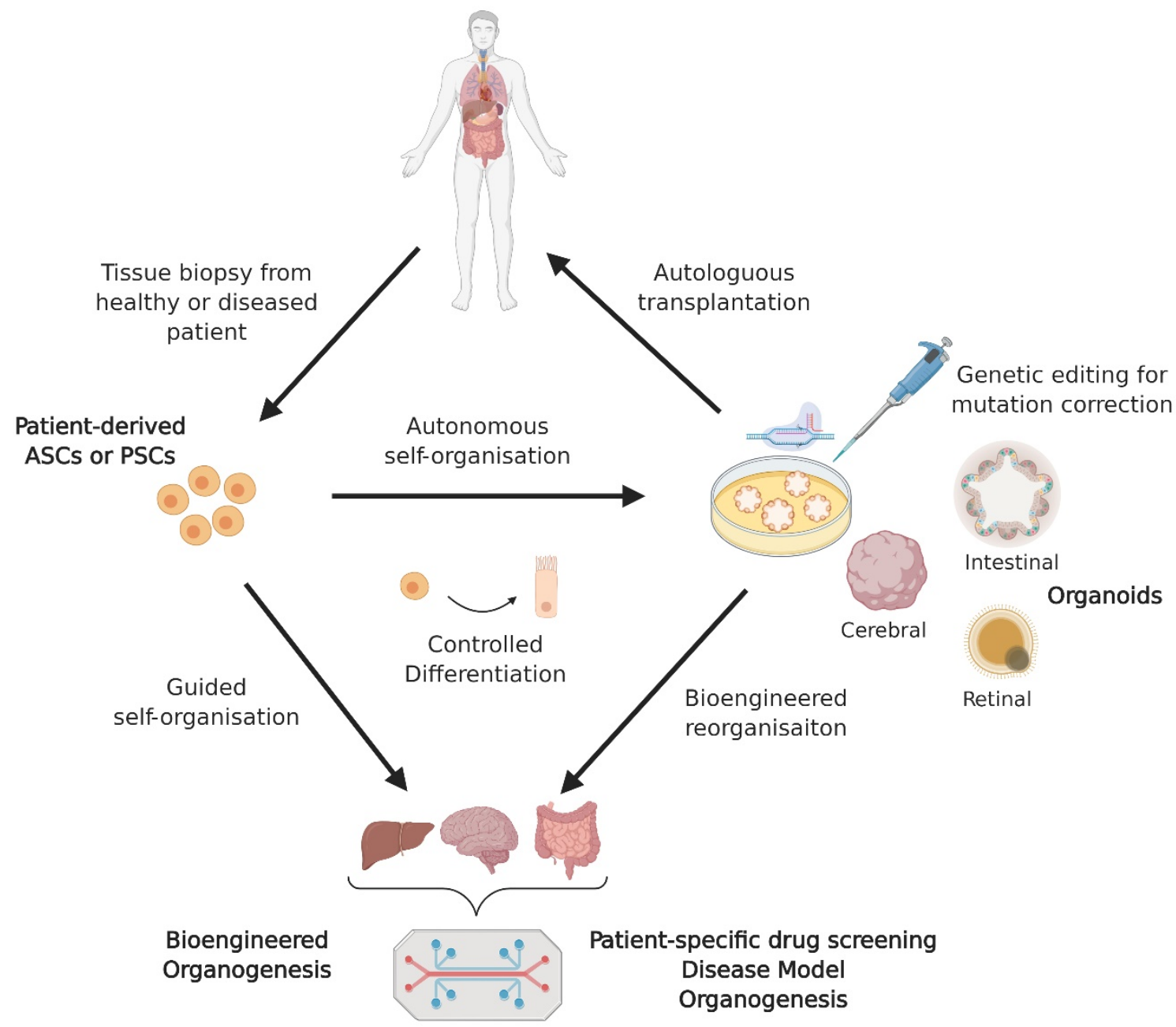

Figure 1: Illustration showing organoid development and different applications of organoids. Organoids can be generated from a variety of organs using adult stem cells (ASCs) or pluripotent stem cells (PSCs). They can be genetically modified to model diseases or correct a mutation from patient-derived stem cells. Bioengineering tools can enhance the physiological relevance of organoids and their utility in drug screening, regenerative therapies, and fundamental biology studies. 

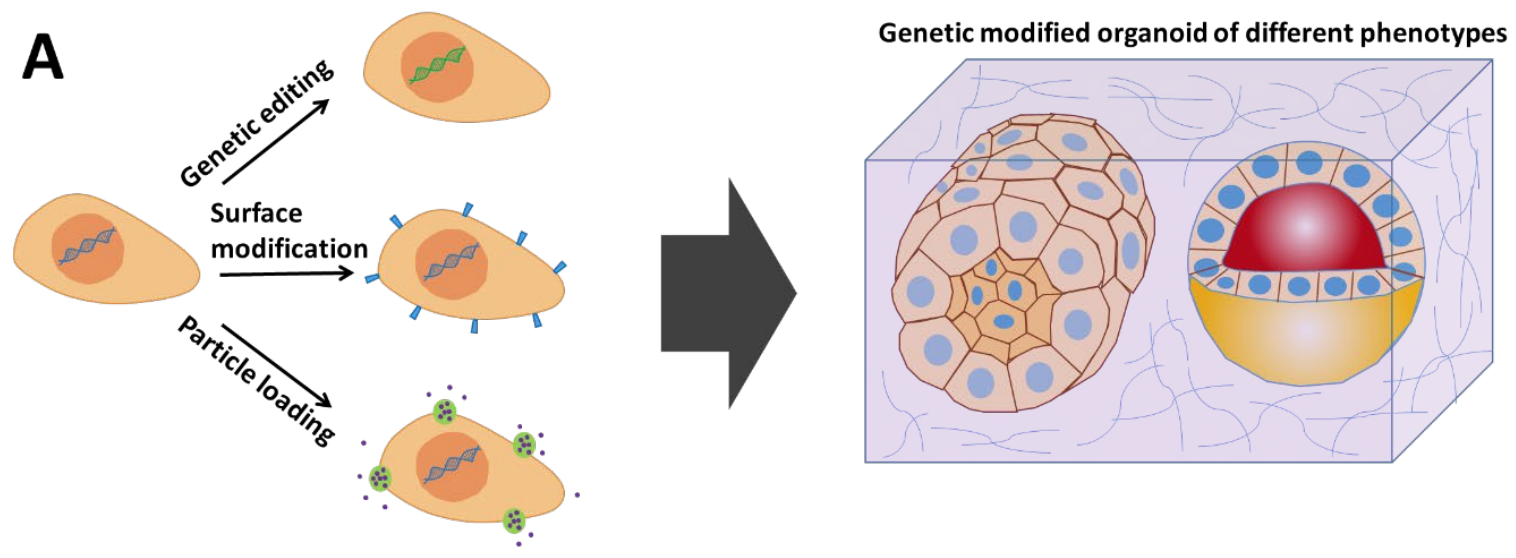

B

Photolithography micropattern

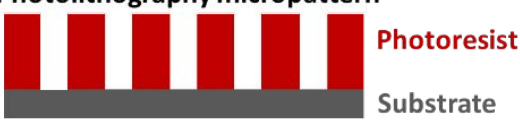

Molding
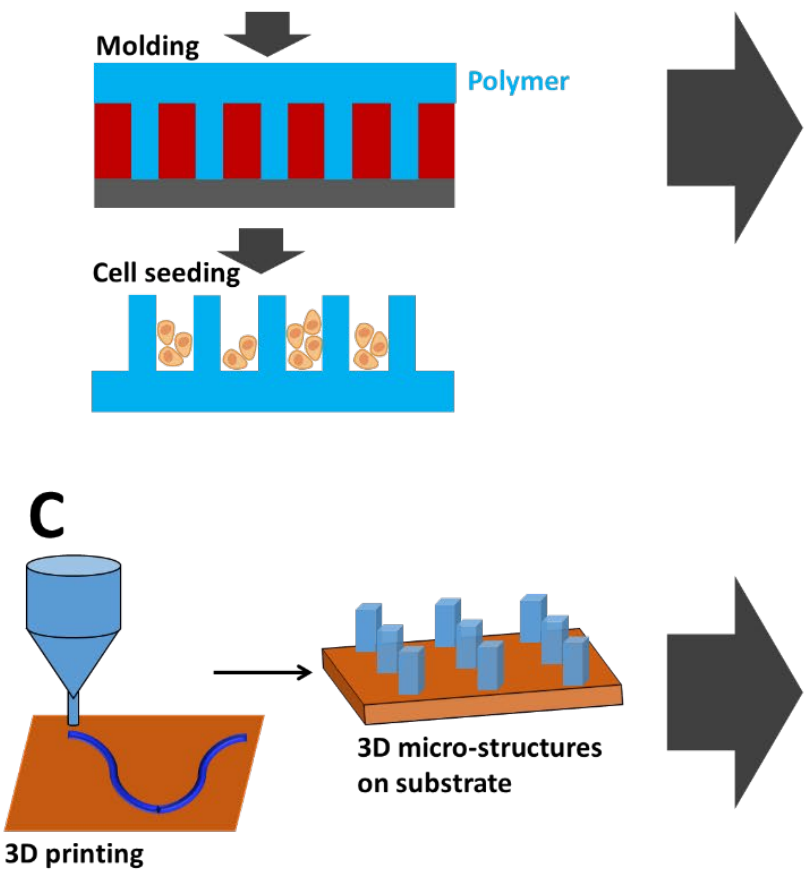

3D printing

Figure 2: Illustration showing various bioengineered approaches for organoid growth and development. (A) Stem cells are genetically modified or their surfaces are conjugated chemically for programmed assembly or loaded with growth factor bearing microbeads for regulating autocrine/paracrine signaling. These methods could generate user-defined organoid phenotypes. (B) Soft lithography can produce 3D confinement, such as micropits, to form initial stem cell aggregates. This approach led to the formation of kidney organoids inside wells of 384-well plates as described by Czerniecki, et al. [2018]. (C) 3D printing fabricates 3D bio-scaffolds to provide physical boundaries as showed by Creff et al. [2019]. 

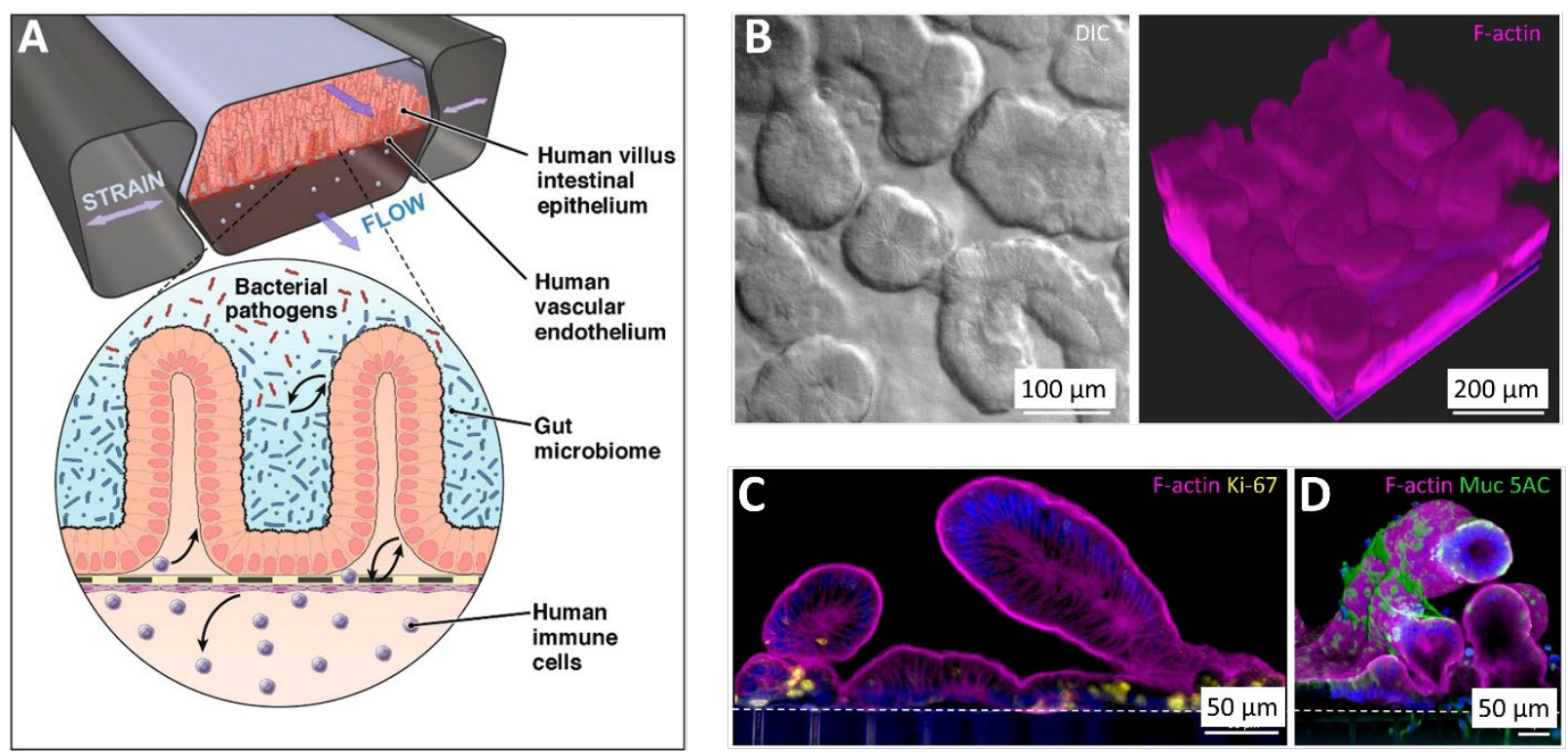

Figure 3: Bioengineered gut-on-a-chip. (A) Illustration of the integration of multiple components into a microfluidic chip for primary human intestinal culture by synergistic bioengineering as described by Bein et al. [2018]. (B) - (D) Morphological analysis of human intestinal organoid-derived epithelia lining the flexible membrane in a gut-chip as demonstrated by Kasendra et al. [2018]. (B) DIC and fluorescent images showing the primary intestinal epithelial layer. (C) and (D) Vertical cross-sections demonstrating villus-like protrusion with condensed brush-border (marked by F-actin), proliferative cells (marked by $\mathrm{Ki}-67$ in (C)) at the basal regions, and mucin-producing cells (marked by Muc 5AC in (D)) along the apical region. 